\title{
The embodied nature of spatial perspective taking: Embodied transformation versus sensorimotor interference
}

Klaus Kessler and Lindsey Anne Thomson

1. Introduction

As a social species, humans are highly skilled in the perception and representation of their conspecifics. This encompasses understanding of simple actions and body postures, such as a hand outstretched for greeting, but also more sophisticated understanding of intentions, such as determining whether somebody is lying or telling the truth. While the former processes have been associated with automatic matching mechanisms without awareness, the latter processes are usually subsumed under the label of "theory of mind" and require conscious understanding of others (see Frith \& Frith, 2007, for a recent review).

In this research we investigated how humans mentally adopt someone else's spatial perspective. While this is a conscious and deliberate process, it is still a quite basic form of inferring other people's representations of the world. Nevertheless it could be an important stepping stone from automatic and unaware perception of others towards more sophisticated forms of 'mind reading'. For instance, similar expressions in several languages use spatial perspective taking as a metaphor for more sophisticated socio-cognitive perspective sharing, e.g. "I understand your point of view", "Put yourself in my position", etc. While this potentially important role in our individual and cultural development remains speculative at this stage, spatial perspective taking (SPT) is an essential process in every day communication and cognition. Consider the following example where we are facing a friend and would like to tell her that there is an eyelash on one of her cheeks (e.g. her left, which would be right from our viewpoint). If we wish to make it easy for our friend then we would mentally place ourselves in her perspective to tell her on which side the eyelash is ("left" in this case). But how do we accomplish such understanding? How do we overcome the differences in body orientations and related perspectives of the world?

\subsection{Spatial perspective taking (SPT) vs. object rotation (OR)}

In fact most people find it quite hard to mentally adopt another viewpoint and research over the past decades has shown that the speed (and accuracy) of SPT decreases with the angular disparity between the egocentric and the target viewpoint (Huttenlocher and Presson, 1973, Kozhevnikov and Hegarty, 2001, Levine et al., 1982 and Zacks and Michelon, 2005, for a recent review). Accordingly, it has been suggested that SPT is subserved by a mental rotation of the self (e.g. Graf, 1994, Keehner et al., 2006, Kessler, 2000, May, 2004, Wraga et al., 2005 and Zacks and Michelon, 2005). In contrast to the ability to mentally rotate objects (OR) (Shepard \& Metzler, 1971), humans seem to adopt somebody else's spatial perspective by mentally rotating themselves into their orientation, which seems to involve a different cognitive operation than object rotation (Hegarty and Waller, 2004, Kozhevnikov and Hegarty, 2001, Kozhevnikov et al., 2006 and Zacks and Michelon, 2005). Kozhevnikov et al. (2006) showed that SPT but not OR performance predicted navigational skills that involved self-to-object relations (e.g. finding short-cuts and pointing to occluded objects). Kozhevnikov and Hegarty (2001) reported a dissociation between the mental abilities for rotating objects versus adopting someone else's perspective although the two processes seemed to be correlated in their setup (also Hegarty \& Waller, 2004). Mental self-rotation has been repeatedly reported to be less effortful (faster/more accurate) than object rotation (OR) within the ground plane (Keehner et al., 2006 and Wraga et al., 1999, for a review; Wraga et al., 2005 and Zacks and Michelon, 2005, for a review) and that discontinuities are observed with SPT but not with OR. That is, processing time for SPT remains fairly constant at low angles but there is a 'jump' around $60^{\circ}-90^{\circ}$ angular disparity where reaction times suddenly start to increase with angle (e.g. Graf, 1994, Keehner et al., 2006, Kozhevnikov and Hegarty, 2001 and Michelon and Zacks, 2006). In contrast, OR shows a continuous increase already at low angular disparities (e.g. Graf, 1994, Keehner et al., 2006, Michelon and Zacks, 2006 and Shepard and Metzler, 1971) but in return seems to dependent less on the plane of rotation (e.g. Zacks \& Michelon, 2005). 
This difference in susceptibility to the plane of rotation suggests that the two processes could be related to different spatial frames of reference. While SPT relies on an egocentric frame, OR implies an allocentric or intrinsic referential frame (Kozhevnikov and Hegarty, 2001, Kozhevnikov et al., 2006 and Wraga et al., 1999). The former encodes object locations in relation to the observer's body orientation, while the latter encodes objects in relation to the environment, i.e. to other objects (and potentially to their intrinsic orientation, e.g. Levelt, 1996). Egocentric encoding could be a first hint towards embodied representations, since the egocentric system has been suggested to be responsible for guiding body movements in space, hence, providing an embodied frame of reference for mental transformations (Kozhevnikov et al., 2006).

\subsection{Motoric embodiment of OR and SPT}

If it was indeed the case that SPT involves some sort of "rotation of the self" then it would be essential to understand what this "self" actually entails. For one branch of the involved research it seems to refer to the transformation of an abstract coordinate system where the observer is basically the point of origin, usually termed "origo" in linguistics and computational linguistics (e.g. Grabowski and Miller, 2000, Graf, 1994, Levelt, 1996, Moratz and Tenbrink, 2006 and Retz-Schmidt, 1988, for a general overview), while on the other side of the spectrum researchers assume that 'mental rotation of the self' involves transformations of the internal representations that the observers possess of themselves (e.g. Arzy, Thut, Mohr, Michel, \& Blanke, 2006; e.g. Blanke et al., 2005, Farrell and Thomson, 1999, Kozhevnikov et al., 2006, May, 2004, Presson and Montello, 1994 and Rieser, 1989). This latter research assumes that SPT is grounded in the internal representations of our body (i.e. body schema) and that the required cognitive transformations are therefore 'embodied'. Note that in the context of SPT adopting another perspective is sometimes termed "disembodiment" since participants have to imagine themselves outside their own body (e.g. Blanke et al., 2005, Klatzky et al., 1998 and Tversky and Hard, 2009). Here we generally term SPT as being embodied - also when adopting another viewpoint - in the sense that we claim (and provide evidence) that SPT is heavily rooted in representations of the body and its movement repertoire. We use the term "embodied" in analogy to "embodied perception" and "embodied semantics" associated with representations partially implemented by the motor and somatosensory system (e.g. Fischer \& Zwaan, 2008).

With respect to embodiment, OR has been shown to be modulated by concurrent movements of the hands (Wohlschlager \& Wohlschlager, 1998). With congruent movements OR is processed faster than with incongruent movements suggesting an overlap between object transformations and action-related representations of hands. Sack, Lindner, and Linden (2007) reported even stronger embodiment of OR in case body parts (hands) had to be mentally rotated. This is in line with the so-called direct-matching hypothesis (Wohlschlager, Gattis, \& Bekkering, 2003) and its assumed implementation by the mirror neuron system (e.g. di Pellegrino et al., 1992, Kessler et al., 2006, Keysers and Perrett, 2004 and Rizzolatti and Craighero, 2004; but see Jonas et al., 2007), which proposes a direct activation of the observer's motor repertoire by the mere observation of an action. For OR this is supported by neuroimaging results where motor areas of the brain were found to be involved during both types of OR, but more strongly during hand- than abstract cubes rotations (e.g. Kosslyn et al., 1998 and Wraga et al., 2003).

Amorim, Isableu, and Jarraya (2006) went a step further in their behavioural experiments and compared OR of abstract cube configurations (cf. Shepard \& Metzler, 1971) to OR of full bodies in various postures. Based on their results Amorim et al. (2006) suggested the notion of motoric embodiment as an integral part of the mental rotation of objects that happen to be bodies. Such motoric embodiment enables a smooth mental rotation of a visually perceived body by emulating the transformation/rotation of the perceived body within the sensorimotor system of the observer. This is in agreement with the direct-matching hypothesis and explains why rotations of bodies are significantly more efficient than rotations of the classic S-M cubes and, importantly, why bodies displaying impossible postures loose this advantage (Amorim et al., 2006).

However, to be able to embody a displayed body posture for rotating it into a target posture one would have to mentally adopt the starting posture to begin with. Amorim et al. $(2006$, p. 344$)$ indeed hint at this pre-stage by stating that the starting posture would have to be emulated (motorically embodied) to begin the rotation process. 
Such posture emulation, however, has been suggested as a form of SPT (cf. Zacks, Mires, Tversky, \& Hazeltine, 2000) where observers mentally rotate/transform their body into the target posture. We therefore expected that SPT in general would incorporate elements of motoric embodiment. This assumption is supported by neuroimaging results that implicated motor and motor-related areas as an integral part of processing during SPT. While Zacks and Michelon (2005) concluded that posterior frontal motor areas are involved in both, object- and self-rotation (see Vogeley et al., 2004, for similar findings re SPT), Wraga et al. (2005) suggested that object rotation was based on motor-representations that reflected manipulation (pre- and primary motor areas), whereas self-rotation was rather based on proprioceptive and perceptual information (fusiform gyrus, insula). Nevertheless, Wraga et al. (2005) also reported supplementary motor area activation during self-rotation, which suggests a certain amount of motor involvement during SPT. Note that while these neuroimaging results reveal task-related activation changes in sensorimotor brain areas, the exact role of such activations during the process of SPT is unclear. Therefore, the embodied nature of SPT still remains speculative and evidence for a direct link between SPT and own and perceived body postures and movements is still largely amiss. We aimed at closing this gap by means of the series of behavioural experiments presented here.

In particular we hypothesised that the postulated motoric embodiment of SPT would involve different body representations than OR, which we tested by comparing Experiments 2 (SPT) and 3 (OR). OR seems to be either related to the internal representation of the hands that humans usually employ to manipulate objects (Carpenter et al., 1999, Kosslyn et al., 1998 and Sack et al., 2007), or in the case of bodies and body parts OR seems to be related to the corresponding posture and movement representations 'mirrored' in the observer (Amorim et al., 2006, Kosslyn et al., 1998, Sack et al., 2007 and Wraga et al., 2003). SPT on the other hand could be related to body representations that are employed during physical alignment of perspectives, i.e. when we actually move/rotate into another point of view. Especially at higher angular disparities such physical perspective changes involve a turn of the whole body and we expected these parts of the body schema to be the basis of SPT.

\subsection{Posture vs. movement emulation during SPT}

This latter consideration also suggests that the notion of posture emulation as the primary embodied mechanism of SPT (as discussed above) could be too closely related to the direct-matching hypothesis, where a visually perceived action or posture is directly emulated within the observer. Such a conception would always rely on exogenous visual input to resonate with the observer's action and posture repertoire. We therefore suggest referring to this form as 'exogenous' motoric embodiment. In contrast we claim that conscious and intentional cognitive processing can rely on embodied transformations that are self-initiated. This could be the emulation of a movement that is already within the repertoire - like rotating the body into a new orientation - which could directly support the cognitive process in question. We propose to refer to this form as 'endogenous' motoric embodiment and suggest that it is the emulation of a movement in contrast to the more perceptually-based 'exogeneous' motoric embodiment referring to the emulation of a visually perceived posture. We further expected SPT to strongly rely on endogenous motoric embodiment since we propose that SPT is the emulation of a body rotation to physically align perspectives.

\subsection{Transformation vs. sensorimotor interference accounts of SPT}

In the context of the spatial updating research the assumption that the body schema is largely involved in SPT has recently even led to a re-interpretation of angular disparity effects in terms of sensorimotor interference (e.g. May, 2004, Riecke et al., 2007, Wang, 2005 and Wraga, 2003).1 According to this account disparity effects do not occur because of an increased cognitive effort of the mental transformation, but instead, are induced by an increasing conflict between the mentally rotated head direction and the available contradictory proprioceptive information (May, 2004). Several findings have been reported to support this notion: Firstly, the updating effort is much reduced if blindfolded participants actually move/rotate into their new orientation and not only imagine the perspective change (Farrell and Thomson, 1999, May and Wartenberg, 1995, Presson and Montello, 1994 and Rieser, 1989; but see Wraga, 2003), thus, suggesting a process that strongly relies on proprioceptive information and on automatic embodied updating (Riecke et al., 2007). Secondly, disorienting participants by turning them in circles until they 
loose their orientation in relation to the environment improves pointing speed and accuracy, suggesting that disorientation relieves participants from interference between imagined and actual orientation (May, 1996).

While these two findings generally support an involvement of sensorimotor representations, a third result imposes a more direct challenge for the transformation account. May (2004) and Wang (2005) employed a spatial updating task where they provided participants in advance with the information about the required perspective change and with enough time for the participants to mentally adopt this perspective prior to the target object being disclosed (to which they had to point from their new perspective). The crucial challenge for the transformation account was that preparation time did not obliterate the effect of angular disparity, which should have been the case as participants were given the time to calculate the transformation in advance, hence, leaving only sensorimotor interference as a possible explanation (May, 2004 and Wang, 2005). Although the experimental manipulations are elegant and the conclusions compelling, we would like to point out that the cognitive load introduced by the number of potential targets in the object arrays has been neglected so far. Our point is that the difficulty for updating an object array is a direct function of the number of objects (Wang et al., 2006). May (2004) and Wang (2005) used quite complex arrays consisting of 4 and 5 objects respectively. If participants would have used their extra time to mentally rotate themselves AND update the object array before knowing the target object they would have had to maintain all 4/5 objects and their updated locations in relation to the rotated self within working memory - which is costly, especially as one must assume that the orientation of the rotated self is maintained in working memory as well. We propose that it was much easier for the participants to either 'do nothing' or conduct SPT only (without updating the 4 or 5 object locations), wait until the target object was indicated, and then update the representation of this specific object. This particular issue can only be resolved by manipulating the number of objects in addition to providing preparation time.

Here we employed a setup with only 2 objects and we manipulated the body schema itself, which allowed comparing the predictions of the transformation and the interference accounts without the potential confound of enhanced working memory load. In contrast to the effective but somewhat coarse disorientation approach (May, 1996) we used different body postures to systematically vary the amount of sensorimotor congruence or conflict in addition to mere angular disparity (Fig. 1B). Since the general evidence for embodiment of SPT is compelling, a 'pure' transformation account in form of an abstract coordinate system transformation (e.g. Retz-Schmidt, 1988) is highly unlikely to be the appropriate approach2. However, if one assumes that the mental self-rotation entails a transformation of parts of the body schema into a virtual body posture in form of a movement emulation (see above), then sensorimotor information should have an influence in addition to a cognitive effort that increases with angular disparity. Accordingly, if SPT primarily transforms body schema representations, then a physical body posture that is already congruent with the direction of mental rotation provides the transformation process with a computational 'head-start' as it is already turned into the correct direction (compare Fig. 1B). 


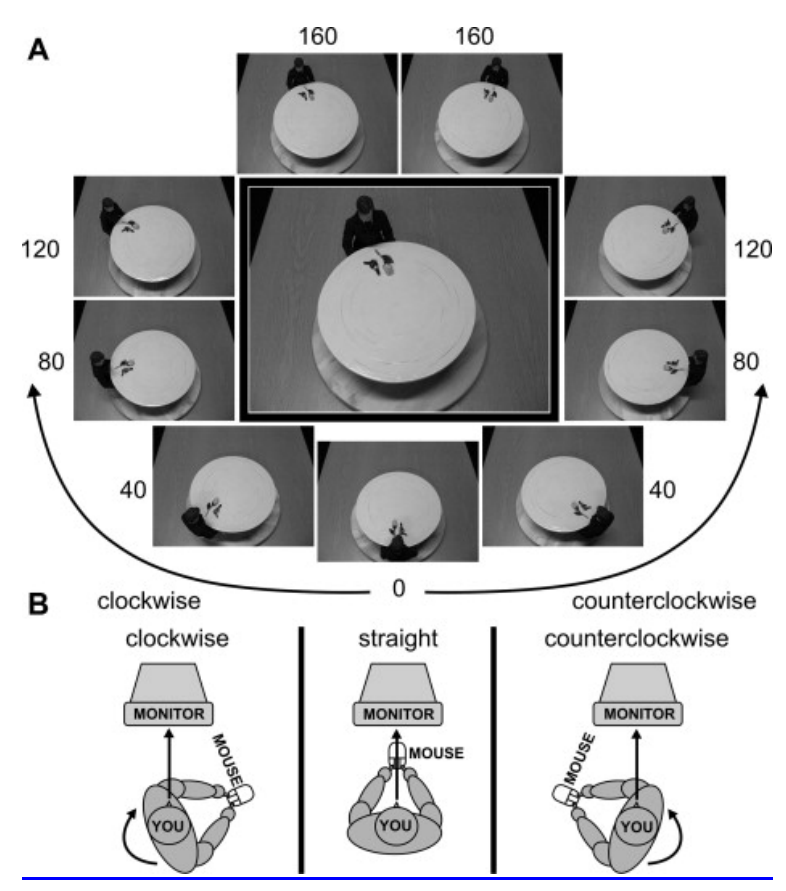

Fig. 1.

(A) Stimuli employed in Experiment 1: the middle picture shows a clockwise rotation of $160^{\circ}$, the surrounding pictures demonstrate all possible rotations. (B) The three possible posture instructions displayed to the participants before each trial.

The difference between the two accounts (sensorimotor interference vs. embodied transformation) now lies in their predictions of how an embodiment effect would change with increasing angular disparity. The embodied transformation account assumes that the congruent body posture provides a 'head-start' which remains constant over angles. That is, the body is already partially turned in the correct direction, thus, decreasing the amount of necessary movement emulation. Since the angle of the participant's physical posture change was constant in all our experiments this head-start or directional priming should always be the same, disregarding the angular disparity for SPT.

In contrast, the interference account predicts a 'best match' effect where the angular disparity that provides the 'best match' between proprioceptive information and mentally transformed perspective should reveal the most efficient processing. In fact the difference between the two accounts boils down to whether sensorimotor congruence/conflict is expected to have a stronger impact than pure angular disparity (sensorimotor interference) or vice versa (embodied transformation) and whether one expects a sensorimotor conflict at the beginning of SPT (embodied transformation) or after (sensorimotor interference).

\subsection{Angular disparity and motoric embodiment}

If SPT was indeed the endogenous emulation of a body rotation then we would expect body posture effects (congruent vs. incongruent) to be optimally revealed when the process of mental self-rotation is actually employed. This seems to be the case when the mental effort for SPT abruptly starts to increase at higher angular disparities. Specifically, Kessler (2000) suggested in concordance with the discontinuities around 60-90 (e.g. Graf, 1994, Keehner et al., 2006, Kozhevnikov and Hegarty, 2001 and Michelon and Zacks, 2006), that a simple visual matching process could be performed at low angles, while actual mental self-rotation commences at angles above $60^{\circ}-90^{\circ}$. This is congruent with Kozhevnikov and Hegarty's (2001) report that for angles below $100^{\circ}$ participants seemed to employ a different processing strategy than SPT, which was reflected by the observation that participants sometimes turned their head to "get a better view" while avoiding to mentally rotate themselves. A visual matching process can be conducted at low angles because the target perspective is still largely aligned with the egocentric perspective. Especially left/right judgements can usually be performed quite easily this way because the target's left and right still 
largely overlap with the observer's left and right - as can be seen in Fig. $1 \mathrm{~A}$ at $40^{\circ}$ angular disparity, where the flower is still clearly left of the gun without a mental self-rotation being necessary. Since we expected that motoric embodiment of SPT would be directly related to the process of mental self-rotation in form of endogenous movement emulation, body posture effects should therefore only appear at higher angles. This still leaves the question open whether sensorimotor congruence/incongruence would have a stronger impact than angular disparity (sensorimotor interference account) or vice versa (embodied transformation account) during mental self-rotation.

\subsection{Research questions}

In a series of four experiments we aimed to reveal whether SPT relies on motoric embodiment. Furthermore we wanted to understand how these results would relate to OR and we expected qualitatively different embodiment patterns for the two processes. We also investigated whether the angular disparity effects in SPT were due to sensorimotor interference (e.g. May, 2004, Riecke et al., 2007, Wang, 2005 and Wraga, 2003) or due to the increasing effort for embodied transformations. We tested an amended form of the basic transformation account which assumes that parts of the body schema serve as the representational basis for the transformation (i.e. embodied transformation account), which in turn is best conceptualised as the self-initiated emulation of a body rotation. In this context we expected motoric embodiment effects to appear at higher angular disparities, strongly depending on whether the process of mental self-rotation would actually be employed to solve the task. Finally we investigated whether SPT would incorporate exogenously triggered posture emulation in addition to self-initiated movement emulation.

\section{Experiment 1}

We aimed to unravel the embodied nature of SPT. To this end we took pictures of an avatar sitting at a round table at various degrees of angular disparity (Fig. 1A). Participants were instructed to adopt the spatial perspective of the avatar and make an object selection from that viewpoint. So far this was a classical setup for a perspective alignment task, where we expected reaction times to increase more strongly at angles over $60^{\circ}-90^{\circ}$ (e.g. Graf, 1994, Keehner et al., 2006, Kozhevnikov and Hegarty, 2001 and Michelon and Zacks, 2006). To test whether the body schema would have an influence on performance, we introduced a novel manipulation: We varied the body posture of the participants (Fig. 1B). Their body posture could either anticipate the direction of mental self-rotation (congruent), or could be in the opposite direction (incongruent), or they remained sitting straight (neutral). Firstly, if SPT was indeed relying on motoric embodiment, then congruent and incongruent postures would enhance or diminish performance, respectively. Secondly, according to the sensorimotor interference account the disparity between the body posture of the participant and the target perspective should have a stronger effect than angular disparity per se, while the embodied transformation account would predict the opposite. We also expected these effects to be observed at higher angles, when mental self-rotation is actually employed.

\subsection{Methods}

\subsubsection{Participants}

In all three experiments participants were volunteers, right-handed, had normal or corrected-to-normal vision, were naive with respect to the purpose of the study, and received payment or course credit for participation. Fourteen females and ten males took part in Experiment 1. Mean age was 21.5 years.

\subsubsection{Stimuli and design}

Visual stimuli showed an avatar sitting at a table at $0^{\circ}, 40^{\circ}, 80^{\circ}, 120^{\circ}$, or $160^{\circ}$, clockwise or counterclockwise, angular deviation (Fig. 1A). Pictures were taken from a vertical angle of $65^{\circ}$. Stimuli were coloured bitmaps with a resolution of 1024 by 768 pixels corresponding to the graphic card settings during the experiment. Viewing distance was $65 \mathrm{~cm}$ and a chin rest was employed to ensure constancy. 
We also varied the body posture of the participants randomly across trials (Fig. 1B). The body in relation to the head/gaze direction could be turned clockwise, counterclockwise or not at all, hence, being congruent, incongruent or neutral in relation to the direction of mental self-rotation. Participants also moved the response device (mouse) together with their body. Marks on the table indicated exactly were to place the mouse to ensure a constant angle of $\pm 60^{\circ}$ (clockwise/counterclockwise) between body and head across trials.

Note that at $0^{\circ}$ angular deviation no mental transformation was required, hence, the straight posture of the participant was most congruent to the task requirements, whereas clockwise and counterclockwise postures were equally incongruent. This implied that the $0^{\circ}$ condition was not included in the MANOVA design, but was assessed in a separate t-test (congruent vs. incongruent).

On every trial a flower and a gun were lying in front of the avatar and participants had to press the corresponding mouse button (left or right) for the side (left or right) on which the target was lying from the avatar's viewpoint. In Fig. $1 \mathrm{~A}$ this would require pressing the left button for the flower or the right button for the gun. The relative positions of the gun and the flower (left/right vs. right/left) as well as the target object (gun vs. flower) were balanced across trials. There was a total of 324 trials.

\subsubsection{Procedure}

Every trial started with the posture instruction (Fig. 1B). When participants had assumed the correct posture they pressed both mouse buttons to proceed to the next step, which was the target instruction. A picture of the target object (gun or flower) was shown together with the respective noun. Participants pressed again both mouse buttons when they felt ready to start the actual task. A fixation cross was shown for $500 \mathrm{~ms}$ and was automatically replaced by the experimental stimulus. Participants were instructed to respond as quickly and as accurately as possible. Audio-visual feedback was then provided reflecting accuracy of the response.

\subsection{Results and discussion}

Since Mauchly's tests revealed that sphericity assumptions were violated in all four Experiments $(p<.05)$, we employed multivariate analyses of variance (MANOVA). In this we followed statistical publications that recommended MANOVA as the method of choice for repeated measures in general ( Davidson, 1972, Obrien and Kaiser, 1985 and Vasey and Thayer, 1987) and in particular when the sample size exceeds the number of levels by at least 10 ( Maxwell \& Delaney, 1990). Two $3 \times 4$ MANOVAs were conducted separately for reaction times (RT; correct responses only) and accuracy data (ACC; percent correct). The repeated measures design consisted of the two factors "body posture" (congruent, incongruent, neutral) and "angle" $\left(40^{\circ}, 80^{\circ}, 120^{\circ}, 160^{\circ}\right)$. As described in Methods, the $0^{\circ}$ condition was analysed in separate t-tests. Partial Eta Squared $\eta_{F}^{2}<$ img height=“21" border=“ 0 " style="vertical-align:bottom" width=" 18 " alt="View the MathML source" title="View the MathML source" src="http://origin-ars.els-cdn.com/content/image/1-s2.0-S0010027709002133-si1.gif"> $\eta$ p2 values will be reported for the main effects as a measure of effect size.

The $3 \times 4$ MANOVA for RTs (Fig. 2A) revealed significant main effects of angle $\left(\mathrm{F}(3,21)=38.3, \mathrm{p}<.001, \eta_{p}^{2}=.846\right)$, body posture $\left(F(2,22)=12.6, \mathrm{p}<.001, \eta_{p}^{2}=.534\right)$, and a significant interaction of angle and body posture $\left(F(6,18)=4.4, \mathrm{p}<.01, \eta_{p}^{2}=.595\right)$. Planned comparisons revealed that a body posture that was congruent to the direction of mental self-rotation was significantly faster than a neutral (straight) posture $(F(1,23)=5.9, p<.05)$, whereas an incongruent posture was significantly slower than a neutral posture $(F(1,23)=9.7, p<.01)$. Accordingly, the congruent was significantly faster than the incongruent posture $(F(1,23)=21.7, p<.001)$. Studentized-NewmanKeuls posthoc tests revealed that RTs significantly increased with angle for all levels of body posture (all $p<.05$ ) except for the increase from $40^{\circ}$ to $80^{\circ}$ in the congruent condition $(p>.1)$. Posthoc tests also revealed that there was no significant difference between any of the three body postures at $40^{\circ}$ of angle (all $p>.1$ ), which fuelled the significant interaction between angle and body posture. The MANOVA for ACC data (percentage correct) revealed a 
main effect of angle $\left(\mathrm{F}(3,21)=4.7, \mathrm{p}<.05, \eta_{z}^{2}=.403\right.$ ), with performance deteriorating with increasing angle ( Fig. 2B).
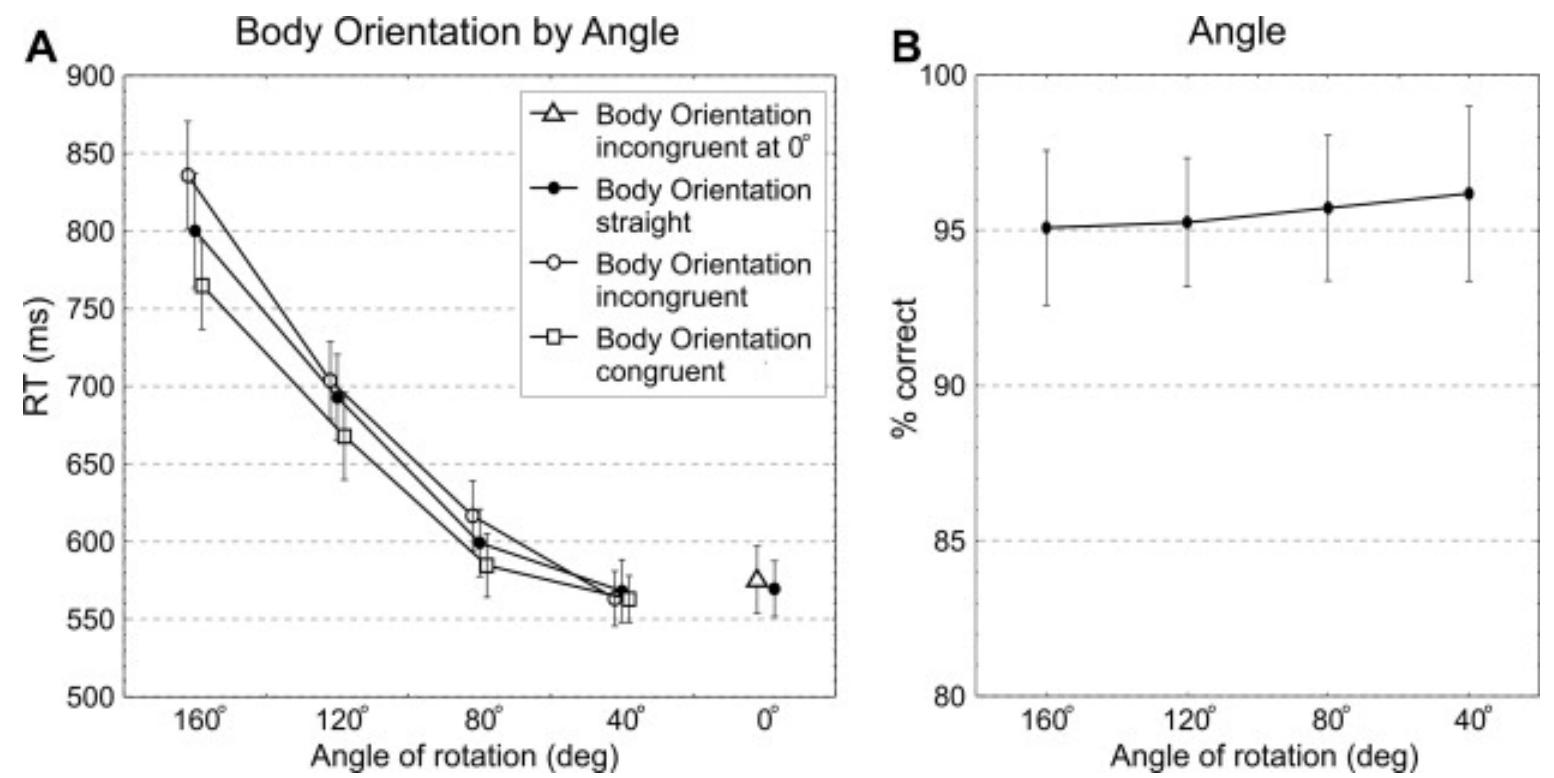

Fig. 2.

Results of Experiment 1. Error bars are standard error of mean. (A) Reaction times (ms) of correct responses as a function of rotation angle and body posture. (B) The main effect of rotation angle for accuracy data (percent correct responses).

The t-tests at $0^{\circ}$ comparing congruent (straight) and incongruent (clockwise + counterclockwise) body postures did not reach significance, neither for RT nor for ACC data (both $p>.1$ ).

\subsubsection{Motoric embodiment}

Besides replicating previous findings showing an increase in the cognitive effort for performing SPT at angles above $40^{\circ}$, we found a robust effect of the congruence between body posture and direction of mental self-rotation. This supports our expectation that SPT is related to the situation-specific body schema of the participants. The significant interaction between angle and body posture suggests that this effect is observed at angles higher than $40^{\circ}$, supporting our claim that motoric embodiment is tied to an increasing need for actually conducting SPT in form of mental self-rotation. Our results suggest a strong motoric embodiment component of SPT, yet, the question remains unresolved whether it is primarily the self-initiated emulation of a body rotation or whether it is mainly the emulation of a visually perceived posture as suggested by Amorim et al. (2006). The simplest way of testing this was to replace the avatar with an empty chair, hence, SPT had to be conducted without a body posture to emulate (cf. Amorim et al., 2006). This manipulation was conducted in Experiment 2, which will be reported after discussing the impact of Experiment 1 on the transformation vs. interference debate.

\subsubsection{Embodied transformation vs. sensorimotor interference}

We observed a clear advantage for congruent over incongruent body postures at angular disparities higher than $40^{\circ}$. At first glance this supports the sensorimotor interference account: proprioceptive information is more similar to the target perspective in the congruent case so generates less interference. However, interference accounts are usually formulated within a head-based frame of reference where the disparity between actual head direction and the tobe-imagined perspective generates the interference (e.g. May, 2004). This will have to be amended to a body-based reference frame as we found congruence effects of the body posture alone without a turn of the head which remained fully aligned with the monitor. 
The difference between the interference and the transformation account with respect to our data lie in their predictions of how the embodiment effect should have changed with increasing angular disparity. The embodied transformation account assumed that the congruent body posture provides a 'head-start' which should result in a constant congruence effect across the higher angular disparities $\left(\geqslant 80^{\circ}\right)$ where self-rotation is actually employed. In contrast, the interference account predicted the strongest congruence effect for the angular disparity where the congruent posture provided the 'best match' while the incongruent posture provided the 'worst match' between proprioceptive information and mental transformation. This is the case at $80^{\circ}$ angular disparity where the $60^{\circ}$ congruently turned body is closest to the target posture (i.e. $\left.-20^{\circ}\right)$, while the incongruently turned body $\left(-60^{\circ}\right)$ is much further away $\left(-140^{\circ}\right)$. This calculation is very different for $160^{\circ}$ disparity, where the congruent body posture now deviates by $(-) 100^{\circ}$ while the incongruent posture deviates again by $(+) 140^{\circ}$. Therefore a much stronger embodiment effect should have been observed for $80^{\circ}$ than for $160^{\circ}$, which however is not the case. The pattern across $80^{\circ}, 120^{\circ}$, and $160^{\circ}$ seems more compatible with a constant head-start effect induced by congruent, neutral, or incongruent proprioceptive information at the start of SPT. An even stronger equivalent conclusion is reached when comparing the RTs of an incongruent body posture at $40^{\circ}$ to a congruent body posture at $160^{\circ} .3$ The deviation between proprioception and target perspective is $100^{\circ}$ in both cases, yet, the RTs for $40^{\circ}$ are much faster than for the large angle $160^{\circ}$ (all $p<.001$ ). This is even more extreme for $80^{\circ}$ where the incongruent condition is still significantly faster (all $p<.001$ ) than the congruent condition at $160^{\circ}$ although the mismatch between proprioception and target posture is actually higher at $80^{\circ} /$ incongruent (mismatch $=140^{\circ}$ ). This contradicts the predictions of the sensorimotor interference account. In conclusion the actual orientation/posture of the body does matter but the transformation of this starting state into the end state matters even more and depends on the angular disparity. Our data suggest that the main conflict is resolved at the beginning of SPT, which is fully compatible with results that show more efficient SPT when proprioception is perturbed (e.g. May, 1996).

In the next experiment we wanted to further consolidate these conclusions while investigating whether the presence of a body (avatar) was essential for the observed motoric embodiment effects during SPT by inducing an emulation of the perceived body posture. Since we proposed that SPT could have evolved from the physical alignment of perspectives (i.e. moving the body into another viewpoint), we believed that the motoric embodiment of SPT might not necessarily depend on the presence of the avatar (posture emulation), as it could mainly represent the self-initiated emulation of a body rotation.

\section{Experiment 2}

In this second experiment we removed the avatar from the scene, replacing it with an empty chair (see Fig. 3). An emulation of a visually perceived body posture was no longer possible. Previous research has clearly shown that SPT can be performed without an avatar being present (e.g. May, 2004 and Michelon and Zacks, 2006), but crucially, would the embodiment effect also persist? If this was the case we would gain novel insights into the nature of the motoric embodiment of SPT. Firstly, it would show that even without a posture to emulate SPT is an instance of motoric embodiment and secondly, depending on the pattern of embodiment effects the results would either further support the embodied transformation account or provide evidence for sensorimotor interference.

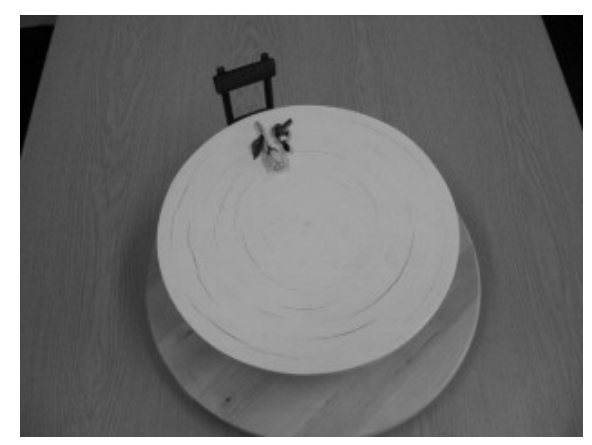

Fig. 3.

Example stimulus in Experiment 2 at $160^{\circ}$ clockwise rotation angle. 


\subsection{Methods}

\subsubsection{Participants}

Twelve female and twelve male volunteers with a mean age of 22.9 years participated in this experiment.

\subsubsection{Stimuli, design, and procedure}

All stimuli, design, and procedure parameters were identical to Experiment 1, only the avatar was replaced by a chair (see Fig. 3).

\subsection{Results and discussion}

The $3 \times 4$ MANOVA on RT data (Fig. 4A) revealed significant main effects of angle $(F(3,21)=16.7, p<.001$, $\left.\eta_{p}^{2}=.705\right)$ and body posture $\left(\mathrm{F}(2,22)=9.9, \mathrm{p}<.001, \eta_{p}^{2}=.473\right)$, as well as a significant interaction between the two factors $\left(F(6,18)=3.9, \mathrm{p}<.02, m_{p}^{2}=.568\right)$. Planned comparisons between the three body postures showed again that a congruent posture was significantly faster than a neutral and an incongruent posture (both $F(1,23)>11.7, p<.01)$, while an incongruent was significantly slower than a neutral posture $(F(1,23)=7.5, p<.02)$. Studentized-Newman-Keuls tests revealed that significant increases in RT related to the angle of rotation occurred only above $80^{\circ} \quad$ (all $p<.05$ ), i.e. for neither body posture a significant increase from $40^{\circ}$ to $80^{\circ}$ was observed (all $p>$.1). Again, body postures did not differ significantly for $40^{\circ}$, yet, also not for $80^{\circ}$ in this experiment (all $p>.1$ ). Taken altogether the effects in Experiment 2 seemed to be even more strongly related to the highest rotation angles $\left(120^{\circ}\right.$ and $\left.160^{\circ}\right)$ than in Experiment 1.
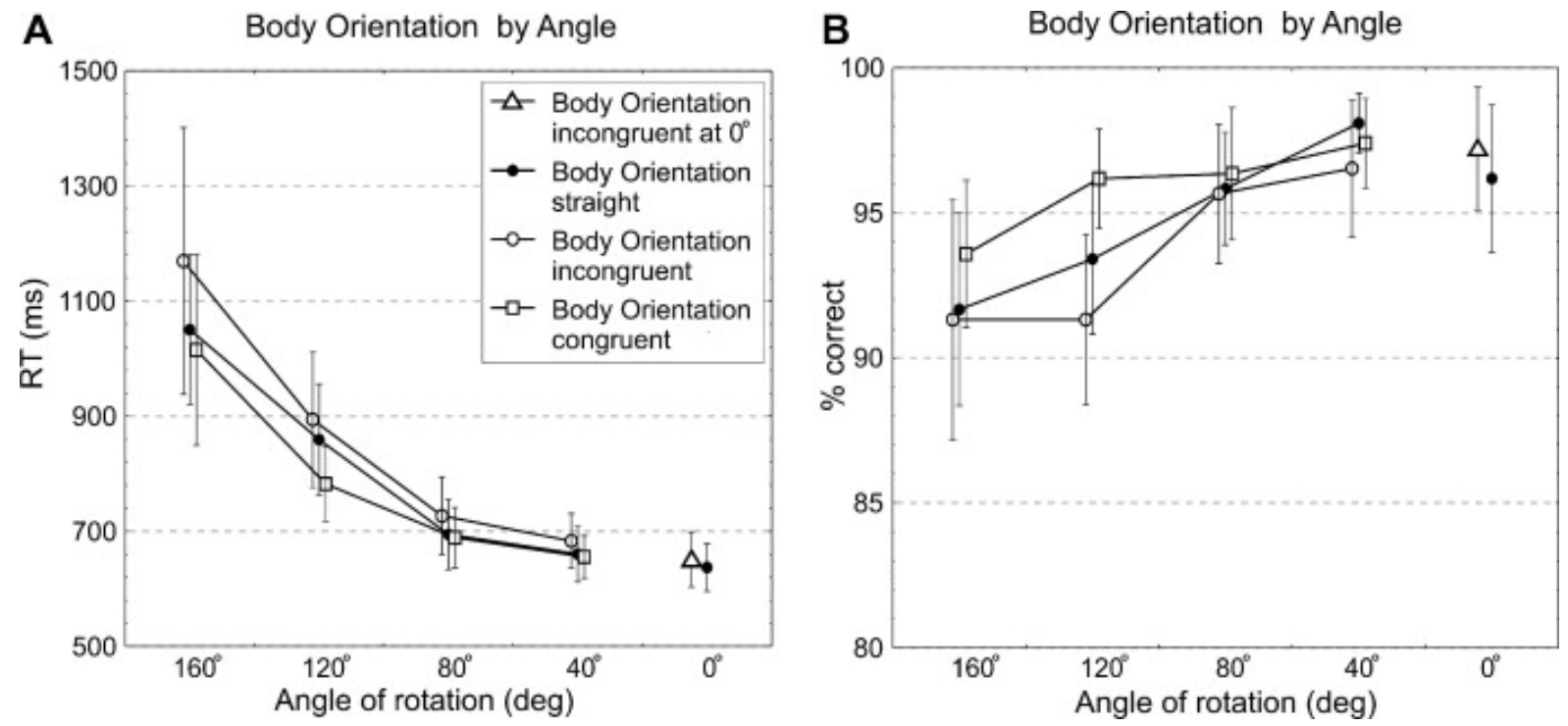

Fig. 4.

Results of Experiment 2. (A) Reaction times (ms) of correct responses as a function of body posture and rotation angle. (B) Accuracy data (percent correct responses) as a function of body posture and rotation angle.

The $3 \times 4$ MANOVA on ACC data (Fig. 4B) revealed significant main effects of angle $(F(3,21)=8.2, p<.001$, $\left.\eta_{p}^{2}=.546\right)$ and body posture $\left(\mathrm{F}(2,22)=6, \mathrm{p}<.01, \eta_{p}^{2}=.354\right)$, while the interaction between the two factors was marginally significant $\left(F(6,18)=2.6, \mathrm{p}<.06, \eta_{p}^{2}=.462\right.$ ). This provided further support for the embodiment effect obtained with RTs. Finally, the t-tests at $0^{\circ}$ rotation angle between congruent (straight) and incongruent (clockwise + counterclockwise) body postures did not reach significance, neither for RT nor for ACC data ( $p>.1)$.

\subsubsection{Embodied transformation vs. sensorimotor interference}


The results of Experiment 2 further corroborate our interpretation of Experiment 1 in that our findings rather support an embodied transformation than a sensorimotor interference account. We observed only a numerical embodiment effect at $80^{\circ} \quad(p>1)$ but a significant effect at $160^{\circ}$. Sensorimotor interference predicted the opposite pattern. Also, RTs at $160^{\circ}$ were generally slower than at $80^{\circ}$ disregarding the participant's body posture (all $\mathrm{p}<.001$ ). Sensorimotor interference predicted faster RTs with a congruent posture at $160^{\circ}$ than with an incongruent posture at $80^{\circ}$. In total the motoric embodiment effect of SPT is strong and reliable but the general transformation effect, i.e. the increase of RTs with angular disparity, was even stronger, which is most compatible with the embodied transformation account.

\subsubsection{Motoric embodiment without an avatar}

Overall Experiment 2 replicated the strong effect of the participant's body posture from Experiment 1. This supports the notion that a significant part of the embodiment effect of SPT is due to a self-initiated emulation of a body rotation without the need for a visually presented body posture to trigger emulation.

However, comparing Fig. 2 and Fig. 4 there seem to be differences between Experiments 1 and 2. Since the design was identical it was possible to directly compare the two experiments in a mixed design MANOVA that included "experiment" as a between groups factor. In addition to significant cross-experimental main effects of body posture $\left(\mathrm{F}(2,45)=16.3, \mathrm{p}<.001, m_{p}^{2}=.42\right)$, angle $\left(\mathrm{F}(3,44)=36.5, \mathrm{p}<.001, \eta_{p}^{2}=.713\right)$, and the significant interaction between body posture and angle $\left(\mathrm{F}(6,41)=3.8, \mathrm{p}<.005, \eta_{m}^{2}=.352\right.$ ), also the interaction between angle and experiment reached significance $\left(F(3,44)=3.2, \mathrm{p}<.05, \eta_{p}^{2}=.181\right.$ ). RTs in Experiment 2 (avatar absent) were increasingly slower with increasing angle than in Experiment 1 (avatar present). This conforms to findings reported by Michelon and Zacks (2006, Experiments 2 vs. 3) who also investigated SPT with and without avatar.

The replication of the embodiment effect in Experiment 2 supports the notion that a significant part of the effect is due to endogenous movement emulation. Yet, the comparison between experiments suggests that omitting the avatar did have an increasingly impeding effect at higher angles. Therefore, the direct test of whether exogenously (perceptually) triggered automatic emulation of a posture modulates SPT in addition (cf. Amorim et al., 2006), was conducted in Experiment 4.

Before answering this more fine-grained question, however, it was necessary to demonstrate the difference in the embodiment of OR versus SPT within our paradigm. This would also confirm that participants did not switch to an OR strategy in the absence of an avatar in Experiment 2.4 As discussed in the Introduction we expected OR and SPT to be embodied in quite different ways. OR (of non-body objects) was reported to be related to representations of the hands (e.g. Wohlschlager \& Wohlschlager, 1998) and not to the whole body like the embodiment effects observed in Experiments 1 and 2. We therefore conducted Experiment 3 where participants were forced to employ OR instead of SPT and we predicted that the participants' posture effect would be obliterated. That is, we wanted to show that the turning of the participants' body only affects SPT and not OR.

\section{Experiment 3}

We aimed to show that the observed motoric embodiment effect is SPT specific and does not occur in the same form in relation to mental object rotations (Shepard \& Metzler, 1971). That is, while OR seems to involve representations of hands which humans usually employ to manipulate objects (Sack et al., 2007 and Wohlschlager and Wohlschlager, 1998), we claim that SPT involves whole body representations that are involved in posture changes to physically align viewpoints.

To investigate OR we employed the stimuli without the avatar from Experiment 2 but changed the task into an object transformation. To this end, the spatial configuration (left/right) of the gun and the flower on the table at various angular deviations was to be matched to the spatial configuration of a red and green block that were always displayed on the table at $0^{\circ}$ (Fig. 5). In order to perform the spatial matching task the two object configurations had 
to be mentally aligned with each other, either by rotating the gun and the flower on top of the red and green block or vice versa. 5 Since this matching task was harder we expected reaction times to increase overall compared to Experiment 2. However, of particular interest here was whether the different body postures would modulate reaction times for object alignment in the same way as for the perspective alignment task (cf. Experiments 1 and 2). Our prediction was that the embodiment effect reflects mental self-rotation and would therefore not be required for object rotation, and hence, no modulation by body posture should be observed in this Experiment 3.

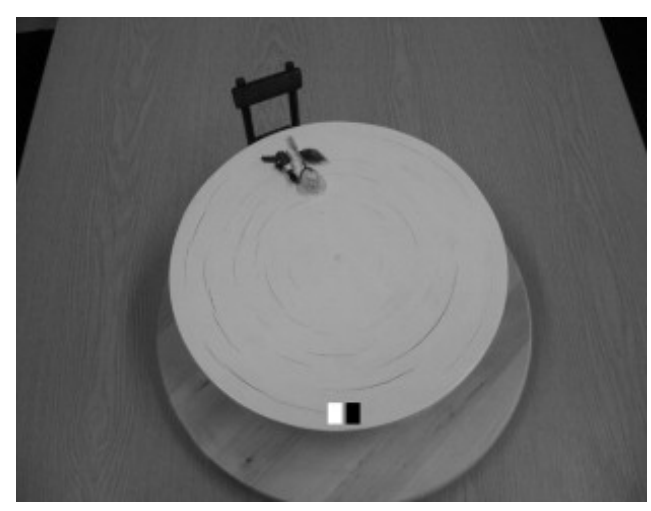

Fig. 5.

Example stimulus in Experiment 3 at $160^{\circ}$ rotation angle. The task was to match the spatial configuration (left/right) of the objects (flower/gun) with the configuration of the two blocks at $0^{\circ}$ by imagining the table to turn until objects and blocks mentally overlapped. Originally the left white block was shown in red and the right black block in green. Flower was always related to the red block whereas gun was always related to the green block, hence, in the example shown here there is a match between object and block configuration. The particulars of mental rotation (rotation of objects versus blocks) was not specified in the instruction.

\subsection{Methods}

\subsubsection{Participants}

Twelve female and twelve male volunteers with a mean age of 23.7 years participated in this experiment.

\subsubsection{Stimuli, design, and procedure}

There were two major changes in Experiment 3, compared to Experiment 2. Firstly, the task was to decide whether the spatial configuration of the flower and the gun (left/right) was matching the configuration of a red and green block (see Fig. 5). For a match the flower had to be in the same relative position as the red block and, reciprocally, the gun as the green block (e.g. if the flower was left of the gun then the red block had to be left of the green block for a match). Secondly, we omitted $0^{\circ}$ rotation angle, since that would have required a direct overlap between the objects and the blocks (Fig. 5).

\subsection{Results and discussion}

The $3 \times 4$ MANOVA on RT data (Fig. $6 A)$ revealed a significant main effect of angle $(F(3,21)=6.8, p<.002$, $\eta_{p}^{2}=.494$ ) but neither of body posture $(F(2,22)<1.48, p>.1)$ nor of body posture by angle $(F(6,18)<1.2, p>.1)$. RTs increased continuously at all angles from $40^{\circ}$ onwards (for all three simple effects, i.e. $160>120>80>40$ : $F(1,23)>4.57, p<0.043$ ). The $3 \times 4$ MANOVA on ACC data ( Fig. $6 B$ ) again revealed a significant main effect of angle $\left(\mathrm{F}(3,21)=8.2, \mathrm{p}<.001, \mathrm{~F}_{p}^{2}=.540\right)$ but neither of body posture $(\mathrm{F}(2,22)<1.48, \mathrm{p}>.1)$ nor of body posture by angle $(F(6,18)<1.2, p>.1)$. 

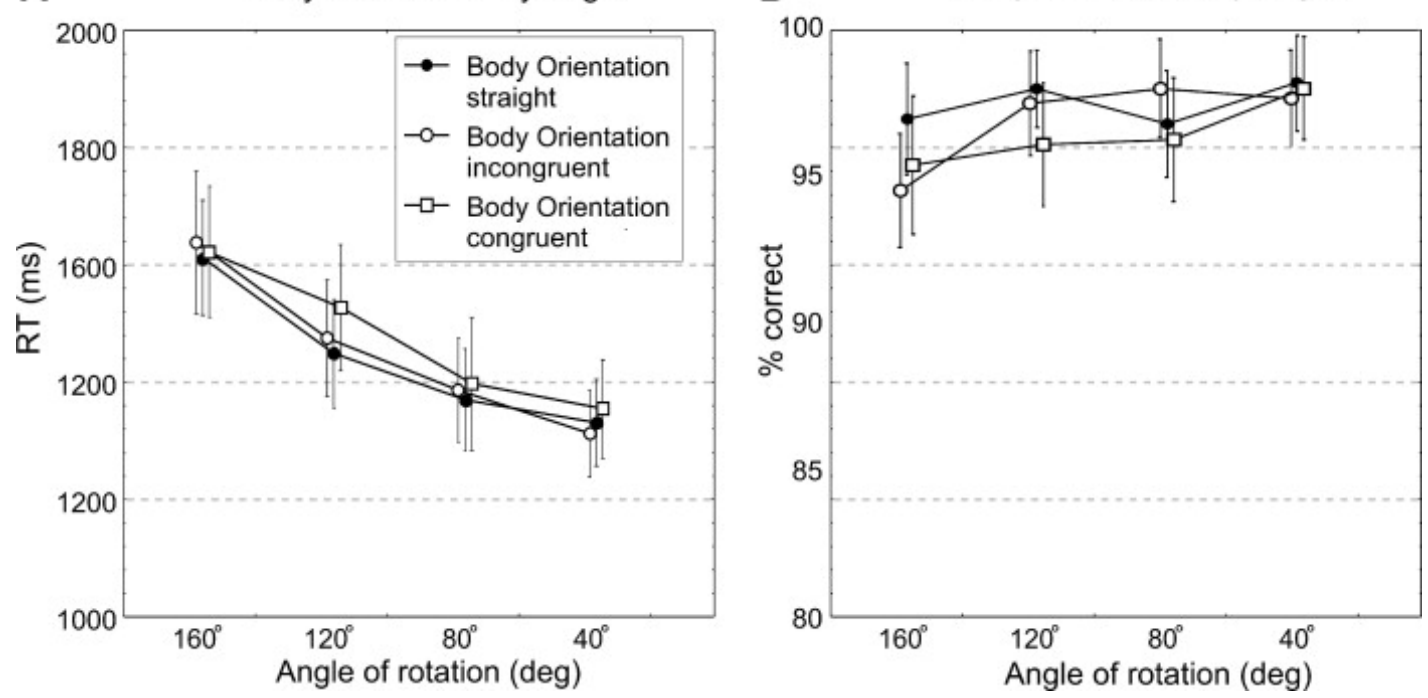

Fig. 6.

Results of Experiment 3. (A) Reaction times (ms) of correct responses as a function of body posture and rotation angle. (B) Accuracy data (percent correct responses) as a function of body posture and rotation angle.

A direct comparison of Experiments 3 and 2 within a mixed design MANOVA $(3 \times 4 \times 2)$ for RT data revealed a significant interaction of body posture and experiment $\left(F(2,45)=8.3, p<.001, \eta_{p}^{2}=.270\right)$ suggesting that the embodiment effect is significantly different between the two Experiments, i.e. present in Experiment 2 and absent in Experiment 3 (compare Fig. 4 and Fig. 6A).

The results allow for the following conclusions. Firstly, the OR Experiment revealed a completely different embodiment pattern (actually none at all) than the SPT Experiments (1 and 2), which shows that SPT is differently embodied than OR. While SPT seems to be related to representations of the whole body, OR (with non-body objects) has been reported to be related to the representations of hands (e.g. Wohlschlager \& Wohlschlager, 1998). In our Experiment 3 we did not systematically manipulate the representation of hands, so we could not replicate the latter finding, but we were able to show in comparison to Experiment 2 that only SPT is related to whole body representations.

Secondly, by confirming that SPT was indeed employed in Experiment 2 even without an avatar, we can make the strong conclusion that SPT predominantly relies on endogenous motoric embodiment in the form of movement emulation and not on exogenously triggered posture emulation. In the final Experiment we therefore resumed our investigations of SPT and aimed to find out whether the motoric embodiment of SPT is completely endogenous, or whether automatically triggered exogenous resonance with a body posture (cf. Amorim et al., 2006) is contributing as well. Although Experiments 1 and 2 overall suggested a major contribution of endogenous movement emulation a first hint that exogenous embodiment could play a role was the finding that omitting the avatar in Experiment 2 did slow down the RTs especially at high angles when self-rotation was employed.

\section{Experiment 4}

As discussed in the context of Experiment 2, we were able to show that motoric embodiment persists in the absence of an avatar, i.e. without the option to match a perceived body onto the internal body schema. We therefore concluded that a large part of the embodiment effect could be related to action emulation (endogenous), but we also pointed out that an additional exogenously triggered effect that would generate a direct match between the perceived body posture and the repertoire of the observer could not be ruled out. We therefore set out to disentangle these two possible sources of motoric embodiment within a single experiment. We re-introduced the avatar but changed the relation between the participant's and the avatar's body postures (Fig. 7). This resulted in two types of congruence: "Movement congruence", which was the congruence employed before, i.e. between the 
participant's body posture and the direction of mental rotation, and "posture congruence", which was the congruence between the body postures of the participant and the avatar (Fig. 7). With these two separate manipulations we were able to disentangle the endogenous (movement emulation) and exogenous (posture emulation) parts of the embodiment effect. Based on Experiment 2 we expected a strong and stable endogenous effect reflected by movement congruence. A significant effect of posture congruence would suggest that exogenous perception-proprioception-matching modulates SPT in addition.

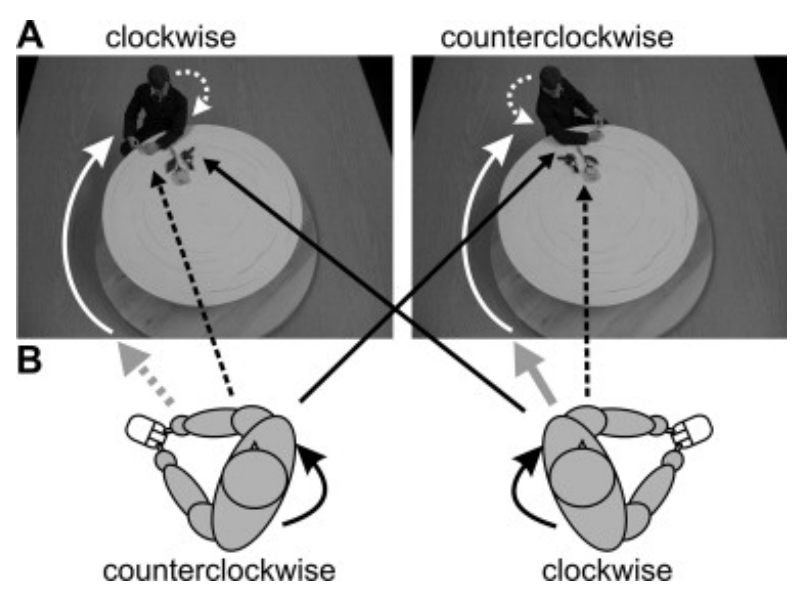

Fig. 7.

Example stimuli in Experiment 4. (A) Both depicted stimuli required clockwise rotation of $160^{\circ}$ (full white arrows), but while the body of the avatar is turned clockwise in the left stimulus, it is turned counterclockwise in the right stimulus (dashed white arrows). (B) The two remaining body postures of the participant (counter- and clockwise). This resulted in two types of congruence: "Movement congruence" between the participant's body posture and the direction of mental rotation (congruent: full grey arrow vs. incongruent: dashed grey arrow), and "posture congruence" between the postures of the participant and the avatar (congruent: full black arrows vs. incongruent: dashed black arrows).

\subsection{Methods}

\subsubsection{Participants}

Twelve female and twelve male volunteers with a mean age of 22.8 years participated in this experiment.

\subsubsection{Stimuli, design, and procedure}

There were three major changes in Experiment 4, compared to Experiment 1. Firstly, the body posture of the avatar could change, inducing posture (in)congruence with the participant's posture (Fig. 5). Secondly, we omitted the straight body posture of the participant (and the avatar) to keep the overall number of trials in a reasonable range. For similar reasons we also omitted the $0^{\circ}$ rotation angle. The total number was 256 trials.

The resulting $4 \times 2 \times 2$ design included three factors: angle $\left(40^{\circ}, 80^{\circ}, 120^{\circ}\right.$, or $\left.160^{\circ}\right)$, movement congruence (congruent or incongruent), and posture congruence (congruent or incongruent). The procedure was identical to Experiments 1 and 2.

\subsection{Results and discussion}

Two separate $4 \times 2 \times 2$ MANOVAs were calculated for RT and ACC data. For RTs (Fig. 8A,B) the MANOVA revealed significant effects of angle $\left(F(3,21)=11.2, p<.001, \eta_{p}^{2}={ }^{* 616}\right)$, movement congruence $(F(1,23)=22.1, p<.001$, $\left.\eta_{F}^{2}=.490\right)$ and the interaction between angle and movement congruence $\left(F(3,21)=3.4, \mathrm{p}<.05, \eta_{F}^{2}=.326\right)$. Studentized-Newman-Keuls tests further showed that the difference between congruent and incongruent trials (movement congruence) reached significance at $120^{\circ}$ and $160^{\circ}$ (both $\mathrm{p}<.01$ ). Posture congruence did not reach 
significance, however, by inspecting Fig. $8 \mathrm{~B}$ a small effect seemed to be present at $120^{\circ}$ and $160^{\circ}$. Accordingly, a simple effect of posture congruence calculated for these two angles reached significance $(F(1,23)=4.4, p<.05)$. This would be quite weak evidence if it was not backed up by the ACC analysis. The MANOVA for the ACC data ( Fig. 8C,D) revealed significant main effects of angle $\left(F(3,21)=8.6, p<.001, \eta_{p}^{2}=.553\right.$, movement- $(F(1,23)=5.7, p<.05$, $\left.\eta_{p}^{2}=.2\right)$, and also posture congruence $\left(\mathrm{F}(1,23)=5.5, \mathrm{p}<.05, \eta_{p}^{2}=.193\right)$.
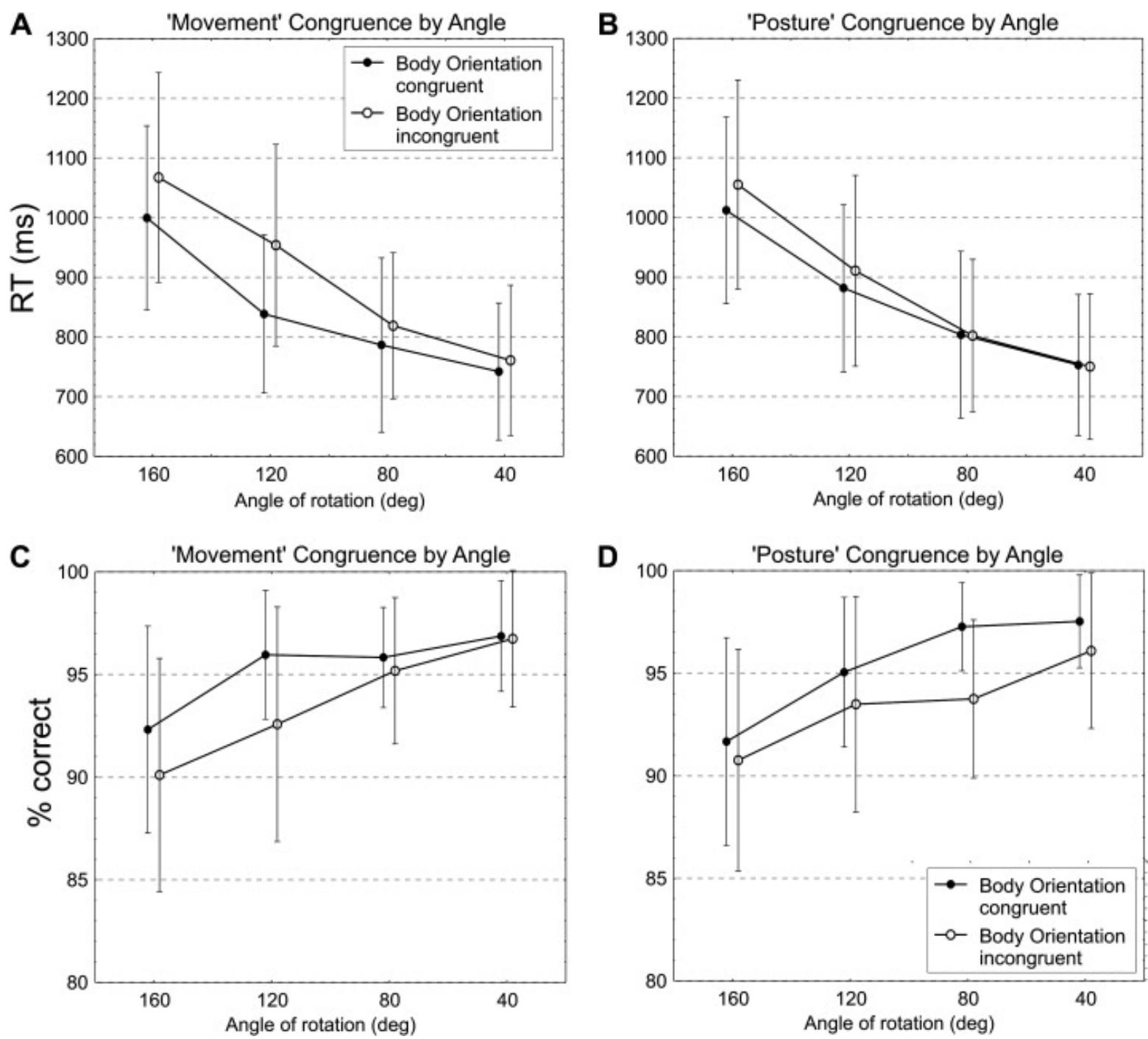

Fig. 8.

Results of Experiment 4. (A) Reaction times (ms) of correct responses as a function of rotation angle and movement congruence. (B) Reaction times as a function of rotation angle and posture congruence. (C) Accuracy data (percent correct responses) as a function of rotation angle and movement congruence. (D) Accuracy data as a function of rotation angle and posture congruence.

The results of Experiment 4 further support our previous findings and suggest that motoric embodiment of SPT is predominantly endogenous, i.e. related to movement emulation. However, we also found evidence that participants could not fully ignore the posture of the avatar, although it was completely irrelevant to the employed objectselection task, suggesting an additional effect of exogenous embodiment based on resonance between the perceived posture and the repertoire of the observer. Conform to Experiments 1 and 2 the pattern of the dominant endogenous effect supports the embodied transformation rather than the sensorimotor interference account by revealing stronger embodiment effects at $160^{\circ}$ than at $80^{\circ}$ and a generally stronger effect of angle (transformation effort) than of body posture (sensorimotor conflict). That is, a congruent posture at $160^{\circ}$ angular disparity was again slower than an incongruent posture at $80^{\circ} \quad(p<.001)$. 


\section{General discussion}

\subsection{Low versus high rotation angles: two mechanisms for SPT}

First of all we were able to replicate previous findings showing an increase in the cognitive effort for performing SPT with increasing angular deviation between the egocentric and the target perspective. We also replicated the classic pattern for object rotation (OR) with a continuous increase of processing time with angular deviation. However, the increase for SPT was not monotonic as effort started to augment significantly above $40^{\circ}$ or even $80^{\circ}$, which is also in agreement with previous findings and suggests two qualitatively different processes for low vs. high angular disparities in SPT (Graf, 1994, Keehner et al., 2006, Kessler, 2000 and Michelon and Zacks, 2006). Kessler (2000) proposed that depending on task particulars the mechanism of mental self-rotation might only be engaged at higher angles since direct visual classification could be possible at low angles. That is, at $0^{\circ}$ deviation participants were able to directly determine which object on the table is left and which is right since the target perspective is congruent to the participants' view of the scene. At $40^{\circ}$ and to a much lesser degree at $80^{\circ}$ this is still possible, as can be observed in Fig. 1: The flower is top-left of the gun at $40^{\circ}$ clockwise, yet still perceivably left. We therefore suggest (cf. Kessler, 2000) that at lower angles, where the relative position of the target objects is largely preserved, responses are fast and accurate as the task may be simply resolved by visual matching. In contrast, at higher angles mental self-rotation becomes necessary.

An important feature of the embodiment effects we found for SPT seems to be that it is confined to these higher angular disparities as reflected by the interaction between angle and participant's body posture in all three perspective alignment experiments (Experiments 1, 2, and 4). In none of these experiments an embodiment effect was observed at $40^{\circ}$. There seems to be a minimum of cognitive effort necessary for congruent - or conflicting information, respectively - to impact on processing speed. We propose that this cognitive effort is imposed by the need for mental self-rotation at higher angular disparities.

\subsection{The embodied nature of SPT}

As our major result we found a robust effect of the congruence between body posture and direction of mental selfrotation in all three experiments on perspective alignment (Experiments 1, 2, and 4). We conclude from these results that SPT essentially comprises an emulation of the sensory consequences (visual and proprioceptive) of a mental rotation of the self, conform to Amorim et al.'s definition of motoric embodiment. Furthermore, we observed this effect with and without an avatar, showing that the emulation process is widely self-initiated in contrast to automatically "mirroring" someone else's body posture (Chatterjee et al., 1996 and Kourtzi and Shiffrar, 1999). At the same time, however, the posture of the avatar could not be fully ignored although it was completely irrelevant to the task (Experiment 4). In this sense we found evidence for motoric embodiment as described by Amorim et al., which we called exogenous (triggered by the observed body posture), but we found even stronger endogenous motoric embodiment in form of a self-initiated emulation of a body rotation. In contrast, the OR task did not reveal any embodiment effect related to the whole body. This is compatible with previous findings showing that OR is strongly related to representations and actions of the hands (Kosslyn et al., 1998, Sack et al., 2007 and Wohlschlager and Wohlschlager, 1998). In that sense SPT and OR are associated with different embodiment effects depending on their affinity to certain parts of the body schema.

While embodied processing could be endogenously initiated or exogenously triggered, proprioceptive representations (body schema) should be involved in any case: We need to "know" our own body posture for either emulating a movement or a posture perceived in others. Accordingly, the neural substrate of SPT prominently seems to consist of parietal regions and areas around the temporo-parietal junction that have been associated with the body schema (e.g. Arzy et al., 2006, Blanke et al., 2005, Keehner et al., 2006 and Zacks and Michelon, 2005).

To re-iterate our data support the view that SPT predominantly relies on the self-initiated emulation of a body rotation. Besides finding a body posture effect without an avatar to emulate in Experiment 2, we disentangled the two possible sources of embodiment in Experiment 4 and found strong and somewhat weaker support for 
endogenous and exogenous embodiment effects, respectively. However, exogenous components of motoric embodiment of SPT could become more important with different tasks; for instance, if the body posture of the target would be more relevant, i.e. by employing an imitation rather than an object-selection task. For example Tversky and Hard (2009) have reported very recently that SPT was conducted spontaneously more often if a person was present in a given scene (corresponding to our avatar) and when queries about spatial relations were phrased in terms of actions.

\subsubsection{Direct-matching versus matching-after-rotation}

The exogenous embodiment component is thought to be related to a direct match between an observed body posture and the internal body schema of the observer (Amorim et al., 2006 and Wohlschlager et al., 2003). Especially at low rotation angles $\left(40^{\circ}\right.$ and $\left.80^{\circ}\right)$ in Experiment 4 a direct match due to congruent postures could actually facilitate processing (Fig. 9A). Although there is no such effect in RTs at low angles, ACC data support this notion (Fig. 8D). In contrast, RTs revealed a subtle effect at $120^{\circ}$ and $160^{\circ}$ (Fig. 8B). Koski, lacoboni, Dubeau, Woods, and Mazziotta (2003) reported that direct-matching between an opposed hand and the imitator's repertoire favours the mirrored hand and not the anatomically corresponding hand (specular imitation, i.e. the actor moves the right hand and the imitator the left hand - as if seen in a mirror). Accordingly, if the simplest form of direct-matching would influence SPT at higher angles, then an incongruent body posture of the avatar should induce the best direct match at $160^{\circ}$, since it would be the (almost) mirrored body posture of the participant ( Fig. 9B). A congruent body posture of the avatar (according to our definition), however, would produce the best match to the participant's posture at $160^{\circ}$ after SPT is completed ( Fig. 9C, compare also Fig. 7). The results are clear and support the latter: a congruent body posture of the avatar speeds up RTs at $120^{\circ}$ and $160^{\circ}$ and is overall less error prone ( Fig. $8 \mathrm{~B}, \mathrm{D}$ ).
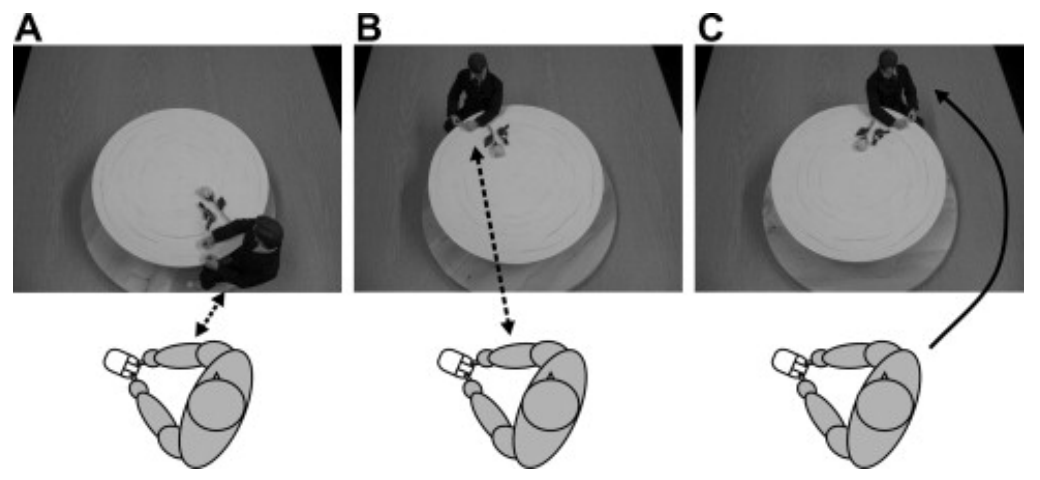

Fig. 9.

Direct-matching (dashed lines) vs. matching-after-rotation (full line). (A) At low angles a direct match between proprioception and the avatar's body posture could facilitate processing. (B) At high angles the avatar almost faces the participant, hence, direct-matching should favour a mirrored body posture (cf. Koski et al., 2003). Note that this is the case for an incongruent posture between avatar and participant. (C) The participant's and the avatar's postures are congruent after SPT, which could facilitate termination of the rotation process along the lines of a matching-after-rotation process. Further explanations in the text.

This leads to the following conclusion. At high angles that are likely to induce a process of self-rotation exogenous embodied processing engages towards the end of the self-rotation process. Possibly, the match between the rotated self and the visually perceived body posture provides a "stop-signal" for the process of rotation, that is when the rotated self perfectly overlaps with, i.e. 'embodies', the target perspective the rotation is terminated. Such a stopsignal would be most efficient if the rotated self and the target perspective match to $100 \%$, that is, when the body postures are congruent. Note that this implies that proprioceptive information about the initial body posture must be rotated as part of the "self", providing further evidence that the "rotating self" during SPT might actually be a transformation of the whole body schema and not simply a rotation of an abstract frame of reference. Accordingly, the absence of an avatar did have a slowing effect at high angles, coinciding with the need for self-rotation (comparing Experiments 1 and 2). This corroborates the notion that the target body posture has an impact on the 
termination of the self-rotation process: incongruent or absent information seems to hamper processing speed. To re-iterate, this also implies that proprioceptive information about the initial body posture is part of the rotating self, further underpinning the conclusion that SPT is the embodied transformation of substantial parts of the body schema.

\subsubsection{Embodied transformation vs. sensorimotor interference}

In all three PT Experiments (1, 2 and 4) we observed a clear advantage for congruent over incongruent body postures at angular disparities higher than $40^{\circ}$. At first glance this supports the interference account: proprioceptive information is more similar to the target perspective in the congruent case so generates less interference. However, in contrast to May's (2004) suggestion, our findings emphasise a body-based over a head-based reference frame, since we found congruence effects of the body posture alone without a turn of the head which remained fully aligned with the monitor.

Furthermore, since the general evidence for embodiment of SPT is quite compelling (e.g. Farrell and Thomson, 1999, May, 1996, May and Wartenberg, 1995, Presson and Montello, 1994 and Rieser, 1989), a 'pure' transformation account in form of an abstract coordinate system transformation (e.g. Retz-Schmidt, 1988, for an overview) was highly unlikely to begin with. Accordingly, if one assumes that SPT entails a transformation of large parts of the body schema into a virtual body posture, then proprioceptive information should have a significant influence in addition to the cognitive transformation effort that increases with angular disparity. We therefore tested the so-called embodied transformation account against the sensorimotor interference account.

At $80^{\circ}$ angular disparity the congruently turned body was closest to the target posture, while the incongruently turned body was furthest away (the difference between the two deviations was $120^{\circ}$ ). Hence, the sensorimotor interference account predicted the strongest embodiment effect at $80^{\circ}$ and a significantly lesser effect at $160^{\circ}$ where the difference between congruent and incongruent body postures in relation to the target perspective was by two thirds smaller (difference was only $40^{\circ}$ ). The results across the three PT experiments are quite clear: in none of the experiments the embodiment effect was larger at $80^{\circ}$ than at $160^{\circ}$ - rather the reverse was the case in Experiment 2. The pattern across $80^{\circ}, 120^{\circ}$, and $160^{\circ}$ in the three PT Experiments is more compatible with a headstart or directional priming effect induced by congruent compared to neutral and incongruent proprioceptive information at the beginning of SPT.

Further support for the embodied transformation account is obtained when comparing the RTs for an incongruent body posture at $80^{\circ}$ to a congruent body posture at $160^{\circ}$. The deviation between proprioception and target perspective is less with a congruent body posture at $160^{\circ}\left(=100^{\circ}\right)$ than with an incongruent at $80^{\circ}\left(=140^{\circ}\right)$, yet, the RTs for $80^{\circ}$ (incongruent) are much faster than for $160^{\circ}$ (congruent) across all three PT Experiments. This strongly contradicts the predictions of the sensorimotor interference account while it is compatible with the notion of embodied transformation.

In conclusion the actual orientation/posture of the observer does matter but the transformation of this starting state into the end state matters even more and depends on the angular disparity. Our data suggest that the main sensorimotor conflict is resolved at the beginning of SPT, when the emulation process of the mental body rotation is initiated. This is fully compatible with results that show more efficient SPT when proprioception is perturbed (e.g. May, 1996). Most importantly, Experiment 4 sheds further light on this issue by supporting the notion that large parts of the body schema are actually transformed during SPT (after the initial conflict has been resolved) as suggested by the accelerating influence of posture matching at the end of the mental self-rotation.

Finally, our findings emphasise the importance of investigating SPT separately from working memory load, or of systematically varying the load and SPT preparation time. As pointed out in the Introduction this could be a possible explanation for why May (2004) and Wang (2005) could not reveal an effect of preparation time, which they interpreted as evidence against a transformation account. However, Wang and colleagues (2006) themselves showed that spatial updating performance strongly depends on the number of objects included in the array. May 
(2004) employed quite complex arrays consisting of 4 objects and Wang (2005) even used 5. If participants would have used their extra time to mentally rotate themselves AND the object array before knowing the target object they would have had to maintain all 4 objects and their updated locations in relation to the rotated self within working memory - which is costly, especially if one assumes that the orientation of the rotated self would have to be maintained in working memory as well. We propose that it was much easier for the participants to wait until the target object was indicated and then update the representation of this specific object. Participants in Wang's (2005) Experiment 2 were instructed to indicate when they had accomplished the perspective change before being told the target. Here again they might have just mentally rotated themselves without updating and costly maintaining the 5 objects in relation to the rotated self, and simply waited for the target to be disclosed. Our prediction would be that with only 1 or 2 objects the extra time would be used indeed for pre-calculating the transformation of the self TOGETHER with the object(s) as the effort for working memory maintenance would be strongly reduced compared to the effort of SPT itself. This particular issue can only be resolved by manipulating the number of objects in addition to providing preparation time. As a first hint, however, Wang et al. (2006) reported a stronger drop in performance with 3 vs. 2 objects than with 2 vs. 1 object. This could point to such a processing dissociation between SPT and updating load with arrays larger than 2 objects.

\subsection{SPT, a stepping stone in evolution?}

The finding that SPT is embodied in form of an emulated movement supports our notion that SPT might have originated from the physical alignment of perspectives by means of actual movements. We therefore suggest SPT as a stepping stone between reflexive control of alignment, e.g. triggered by a gaze cue (Bayliss \& Tipper, 2006), and the conscious mental transformation into an aligned visuo-spatial perspective. Primates (Brauer et al., 2005 and Tomasello et al., 1998) and other species (Brauer et al., 2006, Call et al., 2003, Pack and Herman, 2006 and Scheumann and Call, 2004) have been reported to be capable of simple physical perspective alignment with humans. Primates even change their position to be able to look around obstacles and share the perspective of a human experimenter (Brauer et al., 2005 and Tomasello et al., 1998). While this is not yet SPT it reflects the basic understanding that one has to make a physical (apes) or mental (humans, hominides?) effort to understand someone else's view of the world. Accordingly, Frith and Frith (2007) and Mundy and Newell (2007) have recently argued that sharing our perspective of the world was the starting point for the development of more sophisticated forms of conscious understanding of others. In this sense SPT could mark the transition from responsive physical alignment of attention - available to primates and a few other species - to the conscious and deliberate mental transformation into another perspective of the world - available to humans only (cf. Tomasello, Carpenter, Call, Behne, \& Moll, 2005). At some point of evolution hominids with increased processing capacity might have perfected the technique of adopting the same perspective as a conspecific and thus sharing the view of the world by employing an emulated movement instead of a real one. These origins are still apparent in humans as our research has revealed: not only does SPT appear to be an emulated movement and not a 'pure' rational cognitive transformation, it is also 'accidently' modulated by the displayed body posture, thus, direct matching based on the mirror neuron system that is available to primates as well, still influences this conscious and deliberate cognitive process in humans. This view also conforms to the more radical stance in social psychology, which suggests that the demands of social interaction have in fact shaped perception, action, and cognition (e.g. Knoblich \& Sebanz, 2006).

SPT and therefore embodied processing is indeed involved in high-level conscious and deliberate mental transformations into another perspective of the world. In language, for example, SPT provides an important mechanism for establishing the "common ground" necessary for producing and understanding spatial prepositions like "left" and "right" from various viewpoints other than the egocentric perspective (see Coventry \& Garrod, 2004, chap. 5 for a review; Grabowski and Miller, 2000, Graf, 1994, Kessler, 2000, Levelt, 1996 and Tversky and Hard, 2009). Remember the example in the Introduction: We wish to tell a friend about an eyelash on her cheek. We know we would like to employ a spatial preposition ("left" or "right"), but we have to decide which viewpoint or reference frame to adopt. An egocentric frame of reference would be easier for us, but harder for our friend, and the preposition would be "right" ("you've got an eyelash on the right cheek"). A partner-centred frame of reference would be easier for our friend but harder for us, since we would have to perform SPT to determine the side, and 
hence the corresponding spatial preposition "left" from her viewpoint ("you've got an eyelash on the left cheek"). Depending on the visuo-spatial, yet, also on the social and cultural context ( Coventry and Garrod, 2004, Grabowski and Miller, 2000, Graf, 1994, Kessler, 2000 and Levelt, 1996; see Tversky \& Hard, 2009, particularly for the role of action as context) we might or might not perform SPT, however, as humans we have the choice to deliberately transform our perspective to accommodate constraints of communication and social interaction.

Our research simply points out the 'embodied' origins of these high-level socio-cognitive processes. We predict that the origins of SPT will still influence overt behaviour: for example could we be more inclined to adopt someone else's spatial perspective in a conversation if we happen to have the same body posture (e.g. both sitting cross-legged, arms folded in a chair)? We predict that we will definitely be more inclined towards SPT if our body (but not necessarily the head) is already somewhat turned towards the other person. Could this also be a mechanism for why we perceive others as more 'open-minded', i.e. because they slightly align their body with ours automatically?

Accordingly, our conscious understanding that conspecifics have a different perspective of the world might have also proven essential for other (non-spatial) forms of cognitive perspective taking to evolve like common ground and emulation of the communication partner during language discourse (Barr, 2004, Pickering and Garrod, 2007 and Tversky and Hard, 2009), as well as theory of mind in general (e.g. Frith and Frith, 2007 and Mundy and Newell, 2007). Note, however, that we merely propose that SPT could have been an essential evolutionary stepping stone towards ToM, which introduced a certain concept of thinking about others in the 'easy' spatial domain. This does not necessarily imply that these processes are still implemented by the same cortical networks - although some overlap in executive functions would be plausible.

\section{References}

M.A. Amorim, B. Isableu, M. Jarraya

Embodied spatial transformations: "Body analogy" for the mental rotation of objects

Journal of Experimental Psychology: General, 135 (2006), pp. 327-347

S. Arzy, G. Thut, C. Mohr, C.M. Michel, O. Blanke

Neural basis of embodiment: Distinct contributions of temporoparietal junction and extrastriate body area

Journal of Neuroscience, 26 (2006), pp. 8074-8081

D.J. Barr

Establishing conventional communication systems: Is common knowledge necessary?

Cognitive Science, 28 (2004), pp. 937-962

A.P. Bayliss, S.P. Tipper

Predictive gaze cues and personality judgments: Should eye trust you?

Psychological Science, 17 (2006), pp. 514-520

O. Blanke, C. Mohr, C.M. Michel, A. Pascual-Leone, P. Brugger, M. Seeck et al.

Linking out-of-body experience and self processing to mental own-body imagery at the temporoparietal junction

Journal of Neuroscience, 25 (2005), pp. 550-557

J. Brauer, J. Call, M. Tomasello

All great ape species follow gaze to distant locations and around barriers

Journal of Comparative Psychology, 119 (2005), pp. 145-154

J. Brauer, J. Kaminski, J. Riedel, J. Call, M. Tomasello

Making inferences about the location of hidden food: Social dog, causal ape

Journal of Comparative Psychology, 120 (2006), pp. 38-47 
J. Call, J. Brauer, J. Kaminski, M. Tomasello

Domestic dogs (Canis familiaris) are sensitive to the attentional state of humans

Journal of Comparative Psychology, 117 (2003), pp. 257-263

P.A. Carpenter, M.A. Just, T.A. Keller, W. Eddy, K. Thulborn

Graded functional activation in the visuospatial system with the amount of task demand

Journal of Cognitive Neuroscience, 11 (1999), pp. 9-24

S.H. Chatterjee, J.J. Freyd, M. Shiffrar

Configural processing in the perception of apparent biological motion

Journal of Experimental Psychology - Human Perception and Performance, 22 (1996), pp. 916-929

K.R. Coventry, S.C. Garrod

Saying, seeing and acting: The psychological semantics of spatial prepositions

Psychology Press (2004)

M.L. Davidson

Univariate versus multivariate tests in repeated-measures experiments

Psychological Bulletin, 77 (1972), pp. 446-456

G. di Pellegrino, L. Fadiga, L. Fogassi, V. Gallese, G. Rizzolatti

Understanding motor events: A neurophysiological study

Experimental Brain Research, 91 (1992), pp. 176-180

M.J. Farrell, J.A. Thomson

On-line updating of spatial information during locomotion without vision

Journal of Motor Behavior, 31 (1999), pp. 39-53

Fischer, M. H., \& Zwaan, R. A. (2008). Embodied language: A review of the role of the motorsystem in language comprehension. The Quarterly Journal of Experimental Psychology, iFirst, 1-26.

C.D. Frith, U. Frith

Social cognition in humans

Current Biology, 17 (2007), pp. R724-R732

J. Grabowski, G.A. Miller

Factors affecting the use of dimensional prepositions in German and American English: Object orientation, social context, and prepositional pattern

Journal of Psycholinguistic Research, 29 (2000), pp. 517-553

R. Graf

Self-rotation and spatial reference: The psychology of partner-centred localisations

Peter Lang, Frankfurt (1994)

M. Hegarty, D. Waller

A dissociation between mental rotation and perspective-taking spatial abilities

Intelligence, 32 (2004), pp. 175-191

J. Huttenlocher, C.C. Presson

Mental rotation and the perspective problem

Cognitive Psychology, 4 (1973), pp. 277-299

M. Jonas, H.R. Siebner, K. Biermann-Ruben, K. Kessler, T. Baumer, C. Buchel et al.

Do simple intransitive finger movements consistently activate frontoparietal mirror neuron areas in humans?

Neuroimage, 36 (Suppl. 2) (2007), pp. T44-53

M. Keehner, S.A. Guerin, M.B. Miller, D.J. Turk, M. Hegarty

Modulation of neural activity by angle of rotation during imagined spatial transformations

Neuroimage, 33 (2006), pp. 391-398 


\section{K. Kessler}

Spatial cognition and verbal localisations: A connectionist model for the interpretation of spatial prepositions

Deutscher Universitäts-Verlag, Wiesbaden (2000)

K. Kessler, K. Biermann-Ruben, M. Jonas, H.R. Siebner, T. Baumer, A. Munchau et al.

Investigating the human mirror neuron system by means of cortical synchronization during the imitation of biological movements Neuroimage, 33 (2006), pp. 227-238

\section{Keysers, D.I. Perrett}

Demystifying social cognition: A Hebbian perspective

Trends in Cognitive Sciences, 8 (2004), pp. 501-507

\section{R.L. Klatzky, J.M. Loomis, A.C. Beall, S.S. Chance, R.G. Golledge}

Spatial updating of self-position and orientation during real, imagined, and virtual locomotion

Psychological Science, 9 (1998), pp. 293-298

G. Knoblich, N. Sebanz

The social nature of perception and action

Current Directions in Psychological Science, 15 (2006), pp. 99-105

L. Koski, M. lacoboni, M.C. Dubeau, R.P. Woods, J.C. Mazziotta

Modulation of cortical activity during different imitative behaviors

Journal of Neurophysiology, 89 (2003), pp. 460-471

S.M. Kosslyn, G.J. DiGirolamo, W.L. Thompson, N.M. Alpert

Mental rotation of objects versus hands: Neural mechanisms revealed by positron emission tomography

Psychophysiology, 35 (1998), pp. 151-161

Z. Kourtzi, M. Shiffrar

Dynamic representations of human body movement

Perception, 28 (1999), pp. 49-62

M. Kozhevnikov, M. Hegarty

A dissociation between object manipulation spatial ability and spatial orientation ability

Memory and Cognition, 29 (2001), pp. 745-756

M. Kozhevnikov, M.A. Motes, B. Rasch, O. Blajenkova

Perspective-taking vs. mental rotation transformations and how they predict spatial navigation performance

Applied Cognitive Psychology, 20 (2006), pp. 397-417

W.J.M. Levelt

Perspective taking and ellipsis in spatial descriptions

P. Bloom, M.A. Peterson, L. Nadel, M.F. Garret (Eds.), Language and space, A Bradford Book, Cambridge, MA (1996), pp. 77-108

M. Levine, I.N. Jankovic, M. Palij

Principles of spatial problem solving

Journal of Experimental Psychology: General, 111 (1982), pp. 157-175

S.E. Maxwell, H.D. Delaney

Designing experiments and analyzing data: A model comparison perspective

Wadsworth Pub. Co, Belmont, California (1990)

M. May

Cognitive and embodied modes of spatial imagery

Psychologische Beitrage, 38 (1996), pp. 418-434

M. May

Imaginal perspective switches in remembered environments: Transformation versus interference accounts

Cognitive Psychology, 48 (2004), pp. 163-206 
M. May, F. Wartenberg

Rotations and translations in body-centred space. Models and experiments

Kognitionswissenschaft, 4 (1995), pp. 142-153

P. Michelon, J.M. Zacks

Two kinds of visual perspective taking

Perception and Psychophysics, 68 (2006), pp. 327-337

R. Moratz, T. Tenbrink

Spatial reference in linguistic human-robot interaction: Iterative, empirically supported development of a model of projective relations

Spatial Cognition and Computation, 6 (2006), pp. 63-107

P. Mundy, L. Newell

Attention, joint attention, and social cognition

Current Directions in Psychological Science, 16 (2007), pp. 269-274

\section{R.G. Obrien, M.K. Kaiser}

Manova method for analyzing repeated measures designs - An extensive primer

Psychological Bulletin, 97 (1985), pp. 316-333

A.A. Pack, L.M. Herman

Dolphin social cognition and joint attention: Our current understanding

Aquatic Mammals, 32 (2006), pp. 443-460

M.J. Pickering, S. Garrod

Do people use language production to make predictions during comprehension?

Trends in Cognitive Sciences, 11 (2007), pp. 105-110

C.C. Presson, D.R. Montello

Updating after rotational and translational body movements: Coordinate structure of perspective space

Perception, 23 (1994), pp. 1447-1455

G. Retz-Schmidt

Various views on spatial prepositions

Al Magazine, 9 (1988), pp. 95-105

B.E. Riecke, D.W. Cunningham, H.H. Bulthoff

Spatial updating in virtual reality: The sufficiency of visual information

Psychological Research - Psychologische Forschung, 71 (2007), pp. 298-313

\section{J.J. Rieser}

Access to knowledge of spatial structure at novel points of observation

Journal of Experimental Psychology - Learning Memory and Cognition, 15 (1989), pp. 1157-1165

G. Rizzolatti, L. Craighero

The mirror-neuron system

Annual Review of Neuroscience, 27 (2004), pp. 169-192

A.T. Sack, M. Lindner, D.E. Linden

Object- and direction-specific interference between manual and mental rotation

Perception and Psychophysics, 69 (2007), pp. 1435-1449

M. Scheumann, J. Call

The use of experimenter-given cues by South African fur seals (Arctocephalus pusillus)

Animal Cognition, 7 (2004), pp. 224-230

R.N. Shepard, J. Metzler

Mental rotation of three-dimensional objects

Science, 171 (1971), pp. 701-703 
M. Tomasello, J. Call, B. Hare

Five primate species follow the visual gaze of conspecifics

Animal Behaviour, 55 (1998), pp. 1063-1069

M. Tomasello, M. Carpenter, J. Call, T. Behne, H. Moll

Understanding and sharing intentions: The origins of cultural cognition

Behavioral and Brain Sciences, 28 (2005), pp. 675-691 discussion 691-735

B. Tversky, B.M. Hard

Embodied and disembodied cognition: Spatial perspective-taking

Cognition, 110 (2009), pp. 124-129

M.W. Vasey, J.F. Thayer

The continuing problem of false positives in repeated measures anova in psychophysiology - A multivariate solution

Psychophysiology, 24 (1987), pp. 479-486

K. Vogeley, M. May, A. Ritzl, P. Falkai, K. Zilles, G.R. Fink

Neural correlates of first-person perspective as one constituent of human self-consciousness

Journal of Cognitive Neuroscience, 16 (2004), pp. 817-827

R.F. Wang

Beyond imagination: Perspective change problems revisited

Psicologica, 26 (2005), pp. 25-38

R.X.F. Wang, J.A. Crowell, D.J. Simons, D.E. Irwin, A.F. Kramer, M.S. Ambinder et al.

Spatial updating relies on an egocentric representation of space: Effects of the number of objects

Psychonomic Bulletin and Review, 13 (2006), pp. 281-286

A. Wohlschlager, M. Gattis, H. Bekkering

Action generation and action perception in imitation: An instance of the ideomotor principle

Philosophical Transactions of the Royal Society of London Series B - Biological Sciences, 358 (2003), pp. 501-515

A. Wohlschlager, A. Wohlschlager

Mental and manual rotation

Journal of Experimental Psychology - Human Perception and Performance, 24 (1998), pp. 397-412

\section{Wraga}

Thinking outside the body: An advantage for spatial updating during imagined versus physical self-rotation

Journal of Experimental Psychology - Learning Memory and Cognition, 29 (2003), pp. 993-1005

M. Wraga, S.H. Creem, D.R. Proffitt

The influence of spatial reference frames on imagined object- and viewer rotations

Acta Psychologica (Amsterdam), 102 (1999), pp. 247-264

M. Wraga, J.M. Shephard, J.A. Church, S. Inati, S.M. Kosslyn

Imagined rotations of self versus objects: An fMRI study

Neuropsychologia, 43 (2005), pp. 1351-1361

M. Wraga, W.L. Thompson, N.M. Alpert, S.M. Kosslyn

Implicit transfer of motor strategies in mental rotation

Brain and Cognition, 52 (2003), pp. 135-143

J. Zacks, J. Mires, B. Tversky, E. Hazeltine

Mental spatial transformations of objects and perspective

Spatial Cognition and Computation, 2 (2000), pp. 315-332

J.M. Zacks, P. Michelon

Transformations of visuospatial images

Behavioral and Cognitive Neuroscience Reviews, 4 (2005), pp. 96-118 
1 We would like to thank an anonymous reviewer for emphasising this point.

2 Surprisingly, Amorim et al. (2006, p. 345) claim along similar lines of thought that SPT only involves "spatial" embodiment in contrast to motoric embodiment, which simply assumes an abstract projection of body axes and not motoric posture emulation.

3 We would like to thank an anonymous reviewer for emphasising this point.

4 We would like to thank Maria Kozhevnikov for pointing this out to us.

5 We believe that our small change to the stimuli and the procedure is legitimate to ensure that OR is the only employable strategy - if we would have only changed the instruction the danger would have been great to obtain a mix between OR and SPT depending on each individual's willingness or ability to employ OR (cf. Kozhevnikov et al., 2006, p. 402, 415) in a setup where SPT actually seems to be the easier strategy (Wraga et al., 2005 and Zacks et al., 2003). 